\title{
Conception participative d'innovations et accompagnement des projets d'élevage familiaux au Burkina Faso : explorer, expérimenter et évaluer
}

\author{
Barkwendé Jéthro Delma ${ }^{1}$ Hassan Bismarck Nacro ${ }^{2}$ \\ Valérie Bougouma-Yameogo ${ }^{2}$ Eric Vall $^{3}$
}

\begin{abstract}
Mots-clés
Bovin, conception de projet, exploitation agricole familiale, alimentation des animaux, approche participative, Burkina Faso
\end{abstract}

Submitted: 25 September 2015

Accepted: 11 April 2016

Published: 26 October 2016

\section{Résumé}

Une grande majorité des polyculteurs éleveurs de l'ouest du Burkina Faso ont un projet d'élevage familial (PEF) en tête. Cependant, plus de la moitié des projets ne démarrent même pas. Parmi ceux mis en place, $d$ 'importantes faiblesses sont observées au niveau de la gestion de l'alimentation du bétail, ce qui compromet leur rentabilité économique. Cet article présente une démarche de conception et d'accompagnement de projet d'élevage familial (Capef) conduit en partenariat entre le producteur et un conseiller. Elle permet d'accompagner le producteur dans la préparation, l'exécution et l'évaluation de son projet. La démarche a été élaborée avec 10 producteurs qui avaient des PEF bovins de trait, bovins à l'engraissement ou vaches laitières en préparation. La Capef se déroule en quatre étapes : a) le diagnostic, b) le calibrage et la recherche d'options d'amélioration, c) la mise en œuvre et l'ajustement, et d) l'évaluation. Dans cette approche de conception d'accompagnement, les producteurs ont mis au point le PEF progressivement, en même temps qu'ils ont appris à le piloter, en réorganisant leur travail et l'allocation de leurs moyens de production. La Capef a aidé les producteurs à développer les apprentissages permettant la maîtrise des pratiques d'alimentation et des stratégies d'élaboration d'un projet d'élevage pour mieux le réussir. Pour le chercheur, elle a permis de comprendre la rationalité des éleveurs en participant avec eux à la construction et à la mise en œuvre de leur PEF.

- Pour citer cet article : Delma B.J., Nacro H.B., Bougouma-Yameogo V., Vall E., 2016. Participatory innovation design, and support of family livestock projects in Burkina Faso: explore, experiment, evaluate [in French]. Rev. Elev. Med. Vet. Pays Trop., 69 (2): 63-71

\section{INTRODUCTION}

Dans les pays en développement, des millions d'agriculteurs familiaux souhaitent pratiquer, ou pratiquent déjà, l'élevage pour en tirer un revenu (Coulon et al., 2011 ; Robinson et al., 2011), épargner afin de constituer un patrimoine, se doter d'un « capital - assurance sur pied » en prévision des temps difficiles (Faye, 2001), et disposer d'une source d'énergie agricole et de fumure (Lhoste et al., 2010). Les diverses formes d'élevage familial (bovins de trait, embouche,

1. Cirdes, 01 BP 454, Bobo-Dioulasso, Burkina Faso.

2. IDR, Université polytechnique de Bobo-Dioulasso, Burkina Faso.

3. Cirad, UMR Selmet, 34398 Montpellier, France.

* Auteur pour la correspondance

Tél. : +22670008792 ; email : delmabjethro@yahoo.fr production laitière bovine, élevage de petits ruminants, de porcins ou de volailles) sont présentées comme une option qui aide les producteurs modestes à sortir de la pauvreté en générant rapidement un revenu (FAO, 2009).

En Afrique de l'Ouest l'élevage familial, caractérisé par les liens entre la famille et l'unité de production (inclusion du capital productif dans le patrimoine familial, combinaison de logiques domestiques et d'exploitation) et par la mobilisation du travail familial excluant le salariat permanent (Bélières et al., 2013), est largement dominant face aux formes d'élevage patronales ou industrielles. Aujourd'hui en Afrique de l'Ouest, la croissance démographique, notamment dans les villes, a augmenté la demande en produits animaux de qualité et diversifiés, créant de nouvelles opportunités pour l'élevage familial (Delgado, 2003). Dans la région, cet élevage intégré à l'agriculture est promis à un bel avenir au cours des prochaines décennies (Club du sahel, 2008 ; Robinson et al., 2011). 
De nombreuses études réalisées en Afrique de l'Ouest montrent toutefois que la réussite de ces élevages familiaux généralement de petite taille serait limitée par les crises climatiques, les épizooties récurrentes, la volatilité des prix des intrants d'élevage, la réduction des espaces pastoraux, face auxquelles les producteurs se trouveraient désemparés (Zoundi et al., 2003 ; Sow et al., 2004 ; Bebe et al., 2008). Dans l'ouest du Burkina Faso, plus de $80 \%$ des producteurs ont des projets d'élevage familiaux (PEF) concernant des bovins de trait (BdT), des bovins à l'engraissement (BàE) ou des vaches laitières (VL) (Delma et al., 2016a). Ces PEF ne sont pas innovants, car cela fait longtemps que des producteurs de cette région (même s'ils ne sont pas très nombreux) pratiquent l'embouche bovine, l'alimentation de préparation des animaux de trait avant la campagne agricole et la production laitière. Cependant, plus de la moitié des PEF n'arrivent pas à se concrétiser. Pour les projets réalisés, d'importantes faiblesses ont été relevées concernant notamment l'alimentation du bétail (Delma et al., 2016a).

Aujourd'hui, la demande des polyculteurs éleveurs de l'ouest du Burkina Faso en conception et en accompagnement de leurs projets d'élevage n'est pas couverte dans de très nombreux cas. Pour réussir leurs PEF ils ont besoin d'appui dans le calibrage, l'ajustement et l'évaluation des résultats de ces projets. Pour répondre à cette demande, nous avons élaboré une démarche de conception et d'accompagnement des PEF, basée sur une interaction forte entre le producteur et le chercheur conseiller.

Cet article présente a) la démarche élaborée et testée au Burkina Faso, dénommée « conception et accompagnement des projets d'élevage familiaux en partenariat avec le producteur » (Capef), b) les résultats qu'elle a produits concernant la compréhension du fonctionnement des systèmes d'élevage, et c) les apprentissages qu'elle a induits chez les producteurs qui l'ont mise en œuvre. Les apports et les limites de la démarche seront discutés afin d'identifier les améliorations possibles et son contexte d'utilisation.

\section{MATERIEL ET METHODES}

Létude a été réalisée dans trois villages de la province du Tuy située à l'ouest du Burkina Faso (Gombélédougou, Founzan et Koumbia). La région se caractérise par une prédominance d'exploitations familiales

$\mathrm{PEF}$ : projet d'élevage familial ; BdT : bovins de trait ; BàE : bovins à engraisser ; VL vaches laitières

B : Bwaba; M : Mossi; P : Peulh

UBT : unité de bétail tropical $; 1 \mathrm{UBT}=$ un bovin de $250 \mathrm{~kg}$

$\mathrm{R}$ : PEF en réflexion ; I : PEF initié $;+$ : faible $;++$ : moyenne $;+++$ : forte de polyculture élevage (coton, maïs, bovins). Elle est soumise à un climat de type soudanien (1000 $\mathrm{mm}$ de pluie/an), et présente une forte densité de population (40 à 50 habitants $/ \mathrm{km}^{2}$ ) (Vall et al., 2006). Dans cette zone de nombreuses exploitations ont recours à la traction animale, et l'on trouve de plus en plus d'ateliers de VL et de BàE chez les polyculteurs éleveurs, avec des possibilités d'écoulement des produits vers les villes de Houndé, de Bobo-Dioulasso, voire au-delà, qui ont justifié le choix de cette zone.

Létude s'est appuyée sur 10 PEF portés par des polyculteurs éleveurs dont les caractéristiques principales des exploitations sont présentées dans le tableau I. Ces derniers ont accepté de s'engager dans la démarche Capef pour mûrir et mettre en œuvre trois types de projet : - PEF bovins de trait (trois cas étudiés, $\mathrm{BdT}_{1}$ à $\mathrm{BdT}_{3}$ ), alimentation de préparation des bovins de janvier à mai pour la campagne agricole qui démarre en juin ;

- PEF bovins à l'engraissement (quatre cas étudiés, Bà $\mathrm{E}_{1}$ à $\mathrm{BàE}_{4}$ ), embouche semi-intensive des bovins sur une durée de trois à quatre mois durant la saison sèche :

- PEF vaches laitières (trois cas étudiés, $\mathrm{VL}_{1}$ à $\mathrm{VL}_{3}$ ), production de lait en saison sèche, période de prix élevé du lait.

Les trois PEF bovins de trait étaient portés par des exploitations assez modestes. Concernant les PEF vaches laitières, deux étaient portés par des exploitations disposant d'une bonne capacité financière $\left(\mathrm{VL}_{1}\right.$ et surtout $\mathrm{VL}_{3}$ ). Mais pour $\mathrm{VL}_{3}$ la production de lait était une activité totalement nouvelle. Enfin $\mathrm{VL}_{2}$ était porté par une association de femmes peules qui développait en parallèle une minilaiterie. Sur les quatre PEF bovins à l'engraissement, trois concernaient des exploitations disposant d'une bonne capacité financière ( $\mathrm{Bà}_{1}, \mathrm{Bà}_{2}$ et $\left.\mathrm{Bà}_{4}\right)$ et le troisième impliquait une exploitation plus modeste $\left(\mathrm{Bà}_{3}\right)$.

La démarche Capef a été conçue de manière à améliorer le PEF de façon progressive et incrémentale avec une forte participation du producteur tout au long du processus (préparation, mise en place, évaluation du PEF). Durant la phase de conception du PEF, donc avant sa mise en place, il s'agissait de calibrer et de rechercher des options d'amélioration. Au cours de la phase de mise en place, la démarche visait plutôt l'ajustement du PEF.

La démarche Capef s'est déroulée en quatre étapes (figure 1), de février à mars 2013 pour l'étape de diagnostic des PEF initiaux,

\section{Tableau I}

Caractéristiques des exploitations dans trois villages de l'ouest du Burkina Faso

\begin{tabular}{|c|c|c|c|c|c|c|c|c|c|c|}
\hline \multirow[t]{2}{*}{ Type de PEF } & \multicolumn{3}{|c|}{ BdT } & \multicolumn{4}{|c|}{ BàE } & \multicolumn{3}{|c|}{ VL } \\
\hline & BdT1 & BdT2 & BdT3 & BàE1 & BàE2 & BàE3 & Bà4 & VL1 & VL2 & VL3 \\
\hline Ethnie & B & B & B & M & B & $P$ & $P$ & $P$ & $P$ & B \\
\hline Nb. actifs & 8 & 5 & 3 & 12 & 18 & 7 & 5 & 5 & 11 & 18 \\
\hline Surface cultivée (ha) & 11 & 10 & 11 & 10 & 56 & 1,3 & 7 & 7 & 1 & 56 \\
\hline Nb. UBT bovins & 2 & 3 & 2 & 64 & 98 & 4 & 69 & 69 & 5 & 98 \\
\hline $\mathrm{Nb}$. bovins de trait & 2 & 6 & 2 & 4 & 8 & 4 & 4 & 4 & 0 & 8 \\
\hline Etat du PEF au stade initial & I & 1 & 1 & I & 1 & $\mathrm{R}$ & I & $\mathrm{R}$ & I & $\mathrm{R}$ \\
\hline $\begin{array}{l}\text { Capacité financière } \\
\text { de l'exploitation }\end{array}$ & + & + & + & +++ & +++ & + & ++ & ++ & + & +++ \\
\hline $\begin{array}{l}\text { Expérience du producteur } \\
\text { concernant le PEF }\end{array}$ & Oui & Oui & Oui & Oui & Oui & Oui & Oui & Oui & Oui & Non \\
\hline
\end{tabular}




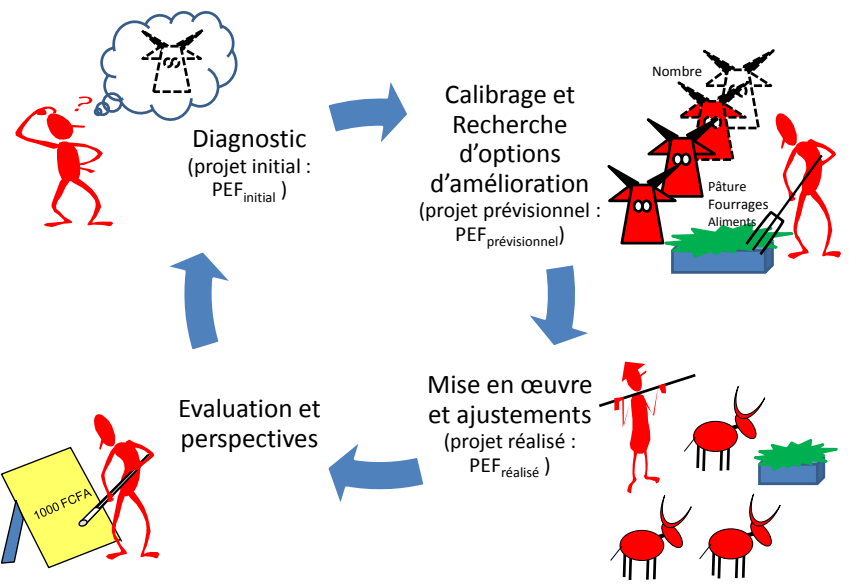

Figure 1 : les quatre principales étapes de la démarche de conception et d'accompagnement d'un projet d'élevage familial (Capef) en partenariat avec le producteur.

d'avril à décembre 2014 pour le calibrage et la recherche d'options d'amélioration des PEF, de janvier à juin 2014 pour la mise en œuvre des PEF et l'ajustement de l'alimentation, avec une étape d'évaluation en fin de processus.

Lors de la première étape (diagnostic), le chercheur a procédé à une analyse du projet initial $\left(\mathrm{PEF}_{\text {initial }}\right)$ en caractérisant l'objectif du producteur, le projet tel que présenté par le producteur (par exemple nombre d'animaux, stocks de fourrages et d'aliments, cultures fourragères), le niveau global de couverture des besoins alimentaires théoriques des animaux pendant la durée du projet, le bilan économique du PEF, afin de cerner les forces et les faiblesses du $\mathrm{PEF}_{\text {initial }}$ La couverture des besoins alimentaires des animaux a été estimée à l'aide d'un calculateur (Altrop) élaboré pour les besoins de l'étude dont les principes sont présentés dans l'encadré 1. Elle a été calculée par le ratio ingéré/besoin, donc exprimée en pourcentage des besoins couverts pour les unités fourragères (UF) et pour les matières azotées digestibles (MAD). Un bilan économique simplifié (recettes dépenses) et un calcul de la marge par animal ont été établis pour les PEF bovins à engraisser et vaches laitières à partir des prévisions de dépenses (entre autres achats d'animaux, valorisation des animaux prélevés dans le troupeau pour les bovins à engraisser, achats de fourrages et d'aliments, soins vétérinaires, dépenses d'équipement) et de recettes (ventes d'animaux, de lait et de produits transformés). Les

Encadré 1

Altrop, UN OUTIL PERMETTANT D'ÉVALUER LA COUVERTURE DES BESOINS ALIMENTAIRES DES BOVINS

Un calculateur, dénommé Altrop (Delma et al., 2016b), a été élaboré avec Excel. Il permet d'ajuster l'offre alimentaire (composée de pâturages, de fourrages récoltés et d'aliments concentrés) proposée à un lot de bovins affectés à une production particulière (énergie agricole, production de viande ou de lait) aux besoins de ces derniers en énergie (UF), en matières azotées digestibles et en sels minéraux (Ca et $\mathrm{P}$ ). Altrop a permis de gérer I'alimentation de lots de bovins en prenant en compte la logique des pratiques d'alimentation des éleveurs, tout en intégrant le système et les références zootechniques du Manuel d'alimentation des ruminants domestiques en milieu tropical (Rivière, 1991)
PEF bovins de trait n'ont pas fait l'objet de cette évaluation car il est difficile d'évaluer une recette ou un gain financier pour ce type de projet.

Durant la seconde étape, le producteur en dialogue avec le chercheur ont recherché des options d'amélioration possibles pour mieux calibrer le projet et pour améliorer la couverture des besoins alimentaires théoriques des animaux. Quand le $\mathrm{PEF}_{\text {initial }}$ avait révélé d'importants déséquilibres alimentaires, des modifications du PEF (durée de pâturage, quantités de fourrages et d'aliments distribuées, ajustement du nombre d'animaux) étaient d'abord discutées avec le producteur. Le calculateur Altrop (encadré 1) permettait de mesurer l'effet des modifications retenues sur la couverture des besoins des animaux et ce résultat était discuté avec le producteur qui validait le changement ou pas. Après plusieurs allers-retours, un projet prévisionnel $\left(\mathrm{PEF}_{\text {prévisionnel }}\right)$ émergeait, dans lequel le nombre d'animaux, les ressources alimentaires mobilisées (notamment stocks d'aliments et de fourrages, introduction de cultures fourragères), la composition des rations et les durées de pâturage avaient évolué. Les incidences des changements étaient enfin prises en compte dans le bilan économique et le calcul de la marge au stade $\mathrm{PEF}_{\text {prévisionnel }}$.

Au cours de la troisième étape, le producteur a mis en œuvre le projet et le chercheur a effectué un suivi du PEF, avec des relevés bimensuels sur les pratiques d'alimentation (temps de pâturage, quantité de fourrages et d'aliments distribués et refusés, évolution du poids et du niveau de production des animaux, entre autres). Le producteur a reçu régulièrement les informations du suivi concernant notamment la couverture des besoins des animaux. Lorsque des déséquilibres importants étaient observés dans les sorties d'Altrop, des ajustements étaient proposés et discutés avec le producteur. Ce suivi a parfois conduit le producteur à ajuster l'alimentation des animaux. Les ajustements étaient aussi pris en compte dans le bilan économique du projet. Au terme du suivi, nous sommes parvenus au projet réalisé $\left(\mathrm{PEF}_{\text {réalisé }}\right)$.

La quatrième et dernière étape a été consacrée au bilan de la Capef pour savoir si les objectifs du producteur avaient été atteints (si non, pourquoi ? et dans ce cas qu'aurait-il fallu faire pour les atteindre ?) et pour discuter avec lui des perspectives qu'il entrevoyait pour l'avenir du projet d'élevage.

Enfin nous avons cherché à savoir si la Capef avait produit des effets sur la capacité des producteurs à gérer un projet d'élevage, ce qui nous a conduits à nous intéresser aux apprentissages induits par la démarche et à évaluer leur profondeur (première ou deuxième boucle ; Argyris et Schön, 2001). Pour les repérer et les évaluer nous avons conduit des entretiens semi-directifs chez les dix producteurs qui ont énuméré et décrit les leçons qu'ils avaient tirées de la démarche. Ces entretiens ont permis de renseigner 65 indicateurs auxquels ont été attribués la valeur 1 lorsque le producteur considérait qu'il s'agissait d'un acquis et 0 dans le cas contraire. Ces indicateurs ont été regroupés en 10 domaines d'apprentissage sur une échelle de 0 à $100 \%$ (moyenne des valeurs des indicateurs rattachés à chaque domaine). L'analyse a permis de comparer les valeurs d'apprentissage selon le stade de réalisation du $\mathrm{PEF}$ (6 $\mathrm{PEF}_{\text {réalisés }}, 4 \mathrm{PEF}$ stoppés au stade $\left.\mathrm{PEF}_{\text {prévisionnel}}\right)$.

\section{RESULTATS}

\section{Etape 1 : diagnostic initial des PEF}

\section{$P E F_{\text {initiaux }}$ bovins de trait}

Dans les $\mathrm{PEF}_{\text {initiaux }}$ bovins de trait (figure 2), l'objectif des producteurs était d'avoir des bovins en bon état corporel pour la campagne agricole qui chaque année débute fin mai. Durant la période d'alimentation de préparation (de janvier à mai), les 


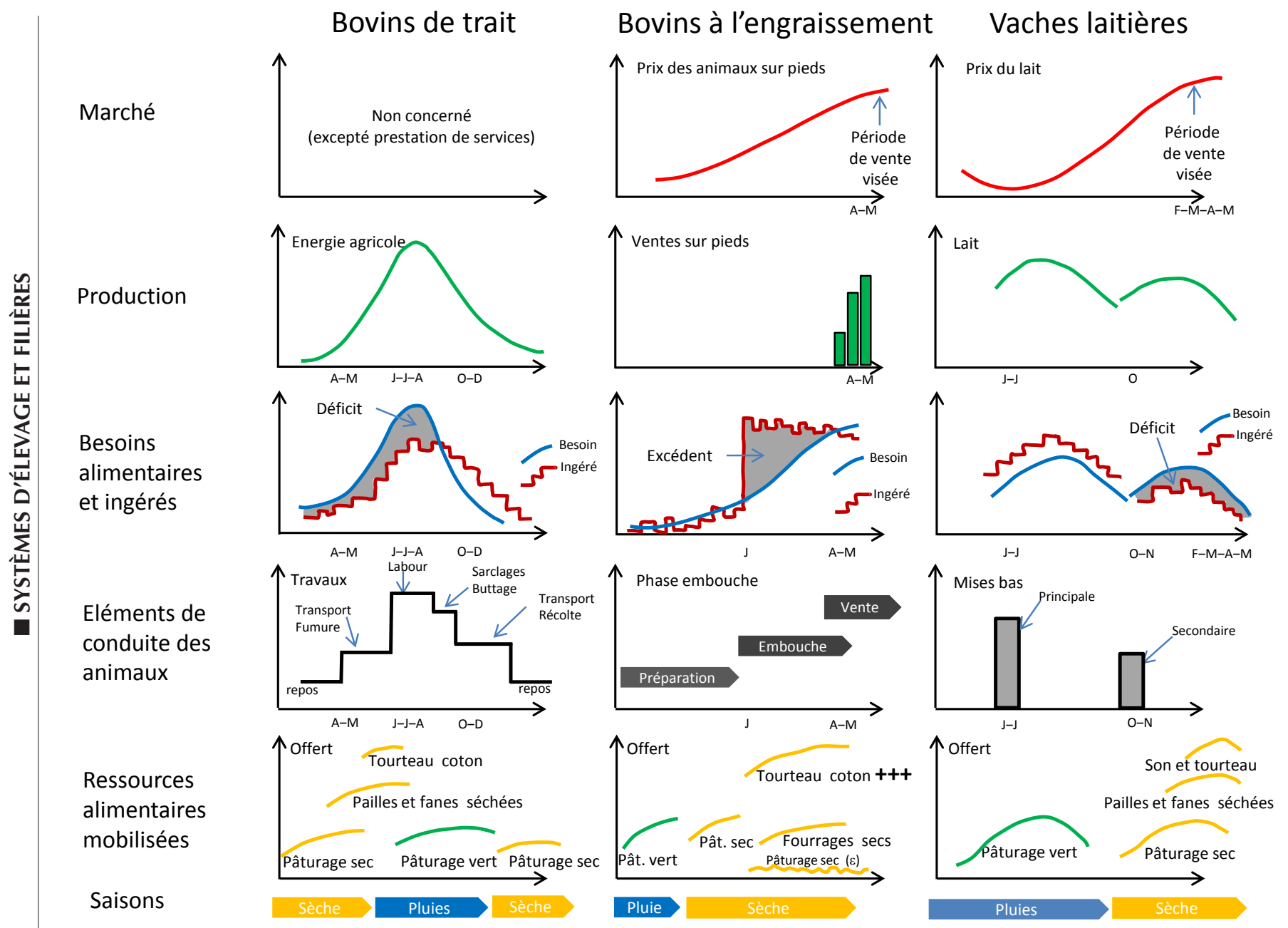

Figure 2 : schématisation des projets d'élevage familiaux. Cette schématisation des trois systèmes d'élevage étudiés dans l'ouest du Burkina Faso a été élaborée à la fin du diagnostic initial à partir des observations et des enquêtes faites sur le terrain, raison pour laquelle les données ne sont pas quantifiées.

bovins étaient d'abord au repos (janvier à mars), les travaux légers reprenaient avec les transports de fumure en avril et les travaux lourds de labour commençaient fin mai. La distribution de fourrages de compléments et d’aliments était prévue à partir de mars ou avril, voire plus tard dans certains cas. Les stocks de fourrage et d'aliments étant faibles à cette période, leur distribution était parcimonieuse.

C'est pourquoi dans la majorité des $\mathrm{PEF}_{\text {initiaux }}$ bovins de trait, les besoins alimentaires des animaux n'étaient pas couverts durant la phase alimentaire de préparation. Sur les trois $\mathrm{PEF}_{\text {initiaux }}$ bovins de trait, deux d'entre eux ( $\mathrm{BdT}_{1}$ et $\mathrm{BdT}_{3}$ ) présentaient un important déficit alimentaire durant cette phase, un seul était en léger déficit $\left(\mathrm{BdT}_{2}\right)$ (tableau II). Le diagnostic a permis de mieux calibrer les projets et d'envisager des options d'amélioration des PEF bovins de trait en vue d'augmenter et de mieux répartir la distribution de fourrage et d'aliments durant la phase de préparation alimentaire.

\section{$P E F_{\text {initiaux }}$ bovins à l'engraissement}

Dans les $\mathrm{PEF}_{\text {initiaux }}$ BàE (figure 2), l'objectif des producteurs était de vendre des bovins engraissés en fin de saison sèche (avril-mai) lorsque le prix du bétail est généralement au plus haut. Ils cherchaient à réaliser une plus-value importante en pratiquant une embouche semi-intensive d'animaux maigres généralement adultes prélevés dans le troupeau ou achetés au marché. Une période de préparation d'un à trois mois, durant laquelle l'éleveur constituait le lot d'animaux à engraisser et administrait des soins sanitaires renforcés aux animaux maigres, précédait la phase d'engraissement qui démarrait généralement en janvier. L'engraissement était prévu sur une période d'environ trois mois, de janvier à mars, durant laquelle les bovins devaient être en stabulation permanente. Durant cette période, les producteurs prévoyaient des distributions quotidiennes de très fortes doses de tourteau de coton et un faible recours au fourrage stocké.

Ce recours massif au tourteau expliquait pourquoi tous les $\mathrm{PEF}_{\text {initiaux }}$ bovins à engraisser présentaient d'importants déséquilibres avec un excès de matières azotées dans les rations par rapport aux besoins théoriques des animaux (tableau II). Cette propension à suralimenter les animaux avec ce concentré disponible localement s'explique par un manque d'informations, l'engraissement étant une activité encore peu répandue dans cette partie du Burkina Faso. L'analyse des $\mathrm{PEF}_{\text {initiaux }}$ bovins à engraisser a aussi mis en lumière des objectifs économiques peu réalistes dans trois projets $\left(\mathrm{BàE}_{2}, \mathrm{BàE}_{3}\right.$ et $\mathrm{BàE}_{4}$ ) (tableau II), présentant des marges prévisionnelles par bovin très élevées, que l'on pouvait aussi expliquer par le manque d'expérience. Le diagnostic a permis de préciser l'objectif de l'étape suivante dans le cas des PEF bovins à l'engraissement : diminuer l'apport de tourteau, renforcer la part des fourrages de qualité dans les rations et réviser les objectifs économiques avec les producteurs peu expérimentés. 


\section{Tableau II}

Evolution des projets d'élevage familiaux dans trois villages de l'ouest du Burkina Faso selon les stades de préparation (initial, prévisionnel) et de réalisation (réalisé)

\begin{tabular}{|c|c|c|c|c|c|c|c|c|c|c|c|}
\hline \multirow[t]{2}{*}{ Type de PEF } & \multirow[t]{2}{*}{ Stade } & \multicolumn{3}{|c|}{ BdT } & \multicolumn{4}{|c|}{ BàE } & \multicolumn{3}{|c|}{ VL } \\
\hline & & BdT1 & $\mathrm{BdT2}$ & BdT3 & BàE1 & BàE2 & BàE3 & Bà4 & VL1 & VL2 & VL3 \\
\hline \multirow[t]{3}{*}{ Nb. d'animaux } & I & 2 & 2 & 2 & 8 & 6 & 4 & 8 & 5 & 5 & 5 \\
\hline & $P$ & 2 & 2 & 2 & 8 & 6 & 4 & 8 & 5 & 5 & 5 \\
\hline & R & 2 & 3 & $x$ & 3 & 5 & $x$ & $x$ & 8 & 3 & 12 à 7 \\
\hline \multirow{3}{*}{$\begin{array}{l}\text { Stock fourrage/ } \\
\text { UBT (kg) }\end{array}$} & I & 374 & 795 & 709 & 544 & 268 & 735 & 716 & 290 & 342 & 101 \\
\hline & $P$ & 1599 & 801 & 709 & 682 & 1619 & 1135 & 856 & 199 & 605 & 252 \\
\hline & $\mathrm{R}$ & 720 & 498 & $x$ & 316 & 54 & $x$ & $x$ & 867 & 139 & 194 \\
\hline \multirow{3}{*}{$\begin{array}{l}\text { Stock aliment/ } \\
\text { UBT }(\mathrm{kg})\end{array}$} & I & 25 & 50 & 50 & 194 & 200 & 125 & 75 & 110 & 90 & 600 \\
\hline & $P$ & 150 & 125 & 75 & 156 & 158 & 50 & 100 & 60 & 70 & 180 \\
\hline & $\mathrm{R}$ & 205 & 133 & $x$ & 216 & 230 & $x$ & $x$ & 657 & 243 & 73 \\
\hline \multirow{3}{*}{$\begin{array}{l}\text { Surface culture } \\
\text { fourragère (ha) }\end{array}$} & I & 0 & 0 & 0,5 & 1 & 0,5 & 0 & 0,75 & 0,75 & 0,25 & 0,5 \\
\hline & $P$ & 0,75 & 1 & 0,5 & 1 & 1 & 1 & 1 & 1 & 0,5 & 1 \\
\hline & $\mathrm{R}$ & 0,75 & 0,75 & $x$ & 0,5 & 1 & $x$ & $x$ & 1 & 0,5 & 1 \\
\hline \multirow{3}{*}{$\begin{array}{l}\text { Couverture besoin } \\
\text { UF/UBT }(\%)\end{array}$} & I & 68 & 83 & 73 & 100 & 74 & 82 & 77 & 81 & 77 & 113 \\
\hline & $P$ & 156 & 119 & 122 & 111 & 190 & 102 & 111 & 71 & 87 & 81 \\
\hline & $\mathrm{R}$ & 115 & 110 & $x$ & 76 & 88 & $x$ & $x$ & 124 & 111 & 97 \\
\hline \multirow{3}{*}{$\begin{array}{l}\text { Couverture besoin } \\
\text { MAD/UBT (\%) }\end{array}$} & I & 70 & 98 & 79 & 357 & 308 & 187 & 165 & 84 & 56 & 121 \\
\hline & $P$ & 216 & 233 & 225 & 259 & 298 & 194 & 245 & 51 & 70 & 63 \\
\hline & $R$ & 151 & 137 & $x$ & 239 & 125 & $x$ & $x$ & 126 & 95 & 70 \\
\hline \multirow{3}{*}{$\begin{array}{l}\text { Marge/UBT } \\
\text { (kFCFA) }\end{array}$} & I & - & - & $x$ & 9 & 246 & 163 & 130 & 51 & 5 & 2 \\
\hline & $P$ & - & - & $x$ & 20 & 253 & 163 & 127 & 51 & 5 & 34 \\
\hline & $\mathrm{R}$ & - & - & $x$ & 45 & 79 & $x$ & $x$ & 21 & 100 & -9 \\
\hline \multirow{3}{*}{$\begin{array}{l}\text { Production de lait } \\
\text { (L/vache/jour) }\end{array}$} & 1 & $x$ & $x$ & $x$ & $x$ & $x$ & $x$ & $x$ & 1,25 & 1,0 & 1,0 \\
\hline & $P$ & $x$ & $x$ & $x$ & $x$ & $x$ & $x$ & $x$ & 1,25 & 1,0 & 1,0 \\
\hline & $\mathrm{R}$ & $x$ & $x$ & $x$ & $x$ & $x$ & $x$ & $x$ & 1,3 & 0,60 & 0,60 \\
\hline
\end{tabular}

PEF : projet d'élevage familial ; BdT : bovins de trait ; BàE : bovins à engraisser ; VL : vaches laitières ;

$\mathrm{I}$ : initial ; P : prévisionnel ; $\mathrm{R}$ : réalisé ; - : non concerné, $\mathrm{x}$ : projet non réalisé

UBT (unité de bétail tropical) $=$ bovin de $250 \mathrm{~kg}$; UF : unité fourragère ; MAD : matières azotées digestibles ; $€=655957$ FCFA

\section{$P E F_{\text {initiaux }}$ vaches laitières}

Dans les $\mathrm{PEF}_{\text {initiaux }}$ vaches laitières (figure 2), l'objectif des producteurs était de vendre du lait durant la deuxième moitié de la saison sèche lorsque le prix de vente du lait est généralement attractif. Mais comme la majorité des mises bas avaient lieu en juin-juillet de l'année précédente et secondairement en octobre-novembre, la production de fin de saison sèche reposait sur une majorité de vaches arrivant en fin de lactation. De plus les stocks de fourrage et d'aliments concentrés destinés à couvrir les besoins des vaches durant la saison sèche étaient souvent trop faibles.

Sur les trois $\mathrm{PEF}_{\text {initiaux }}$ vaches laitières, deux d'entre eux $\left(\mathrm{VL}_{1}\right.$ et surtout $\mathrm{VL}_{2}$ ) ont présenté un important déficit de couverture des besoins des vaches en saison sèche dû à une insuffisance des rations de fourrage $\left(\mathrm{VL}_{1}\right.$ et $\left.\mathrm{VL}_{2}\right)$ et à une insuffisance des doses d'aliments concentrés $\left(\mathrm{VL}_{2}\right)$ (tableau II). Le troisième a présenté au contraire un excédent de matières azotées dû à des rations de tourteau de coton trop importantes comme dans le cas de l'embouche $\left(\mathrm{VL}_{3}\right)$. Sur le plan économique, le PEF porté par la coopérative féminine $\left(\mathrm{VL}_{1}\right)$ a présenté une marge par vache nettement plus importante par rapport aux deux autres parce qu'il prévoyait la transformation du lait brut en yaourt, ce qui n'était pas le cas des deux autres projets $\left(\mathrm{VL}_{2}\right.$ et $\left.\mathrm{VL}_{3}\right)$ où les producteurs se limitaient à la vente de lait brut. Le diagnostic a permis de préciser l'objectif de l'étape suivante dans le cas des PEF vaches laitières : augmenter les quantités de fourrage et d'aliments concentrés dans les projets $\mathrm{VL}_{1}$ et $\mathrm{VL}_{2}$, et réduire les doses de tourteau et ajuster les rations avec des fourrages dans le projet $\mathrm{VL}_{3}$.

\section{Etape 2 : calibrage et recherche d'options d'amélioration des PEF}

A partir des faiblesses des $\mathrm{PEF}_{\text {initiaux }}$ observées lors de l'étape précédente, les discussions entre le producteur et le chercheur ont conduit à modifier progressivement les plans de rationnement des animaux et parfois le nombre d'animaux concernés en vue d'améliorer la couverture des besoins alimentaires des animaux (réduction des déficits ou des excédents selon les cas). Ces ajustements ont eu des répercussions sur les bilans de trésorerie. Au terme de cette étape, les changements 
validés par le producteur ont été intégrés dans le $\mathrm{PEF}_{\text {initial }}$ pour aboutir au $\mathrm{PEF}_{\text {prévisionnel }}$ (projet prévisionnel que l'agriculteur comptait mettre en place).

Une option fréquemment discutée avec les producteurs a été le recours aux cultures fourragères en complément (PEF bovins de trait et vaches laitières) ou en substitution (PEF bovins à engraisser) des aliments achetés. Compte tenu de la disponibilité locale des semences, trois options ont été proposées : le niébé fourrager (Vigna unguiculata), le mucuna (Mucuna deeringiana), le sorgho Grinkan (sorgho hybride). En fonction de la disponibilité en terre et des besoins des PEF, les producteurs ont planifié dans les $\mathrm{PEF}_{\text {prévisionnels }}$ l'installation de surfaces fourragères, plus importantes que dans les $\mathrm{PEF}_{\text {initiaux }}$, entre 0,25 et 2 hectares selon les cas (tableau II), avec une préférence pour le niébé fourrager en raison de son caractère multiusage (production de graines alimentaires et de fourrage).

\section{$P E F_{\text {prévisionnels }}$ bovins de trait}

Dans les PEF bovins de trait, l'intégration de cultures fourragères et d'aliments pour le bétail artisanaux (drêche de dolo et son de céréale disponible au sein de l'exploitation) ont permis d'améliorer les stocks de fourrage et d'aliments, et donc de la couverture des besoins (tableau II) et de réduire les coûts ${ }^{4}$.

\section{$P E F_{\text {prévisionnels }}$ bovins à l'engraissement}

Dans les PEF bovins à engraisser, les ajustements des rations alimentaires discutés avec les producteurs (réduction des doses de tourteau compensée par du fourrage) ont permis de réduire un peu les excès de matières azotées et de rééquilibrer les apports en énergie sans toutefois parvenir à l'équilibre, parce que les stocks prévisionnels de fourrage envisagés par les producteurs n'étaient pas suffisants pour effectuer un bon rééquilibrage (tableau II). Toutefois, ces ajustements ont permis d'améliorer le bilan économique au moins dans un cas $\left(\mathrm{Bà}_{1}\right)$ et ne l'ont pas affecté dans les autres cas.

$P E F_{\text {prévisionnels }}$ vaches laitières

Dans les PEF vaches laitières les ajustements des rations alimentaires discutées avec les producteurs (tableau II) ont permis de réduire un peu le déficit alimentaire dans un seul cas $\left(\mathrm{VL}_{2}\right)$, grâce à la production de fourrage et à l'accroissement des stocks de résidus agricoles. Dans les deux autres cas les réductions de recours aux aliments ont été insuffisamment compensées par du fourrage de qualité, ce qui explique un creusement du déficit dans les $\mathrm{PEF}_{\text {prévisionnels }}$ de $\mathrm{VL}_{1}$ et $\mathrm{VL}_{3}$. Chez ce dernier, la réduction drastique du recours au tourteau a expliqué l'amélioration de la marge. Le creusement du déficit au stade prévisionnel chez $\mathrm{VL}_{1}$ et $\mathrm{VL}_{3}$ traduit des moments d'incompréhension entre le conseiller et le producteur sur la façon de planifier l'alimentation et montre la difficulté du conseil.

\section{Etapes 3 et 4 : mise en ouvre des projets et ajustements effectués et bilan}

\section{Mise en auvre effective des projets}

Après examen du projet initial et définition des mesures d'ajustement, sept producteurs ont choisi de mettre en œuvre leur PEF, et trois ont préféré abandonner ou ont reporté la mise en œuvre à une date ultérieure. Les principales raisons évoquées par les producteurs pour justifier l'abandon ou le report étaient : a) des difficultés pour réunir les moyens financiers pour l'achat des animaux pour l'embouche et b)

\footnotetext{
4 Tarifs de quelques aliments et coût de production des fourrages (nos estimations) : tourteau de coton $(120 \mathrm{~F} / \mathrm{kg})$, son de maïs $(60 \mathrm{~F} / \mathrm{kg})$, farine basse de riz $(20 \mathrm{~F} / \mathrm{kg})$, drêche de dolo $(65 \mathrm{~F} / \mathrm{kg})$, coût de production fanes de niébé et de
} mucuna $(60 \mathrm{~F} / \mathrm{kg})$. une production fourragère insuffisante pour couvrir les besoins des animaux. On peut toutefois ajouter que l'examen des projets a aussi permis de mettre en évidence dans ces cas, d'une part, un manque de réalisme dans l'élaboration des projets avec des coûts sous-évalués et des profits escomptés surestimés et, d'autre part, une stratégie de commercialisation mal établie.

\section{Ajustements des PEF et bilan}

\section{- Ajustements des PEF bovins de trait}

Dans les PEF bovins de trait réalisés, l'offre en aliments a été augmentée, les surfaces de cultures fourragères prévues à peu près réalisées et dans un cas au moins $\left(\mathrm{BdT}_{1}\right)$ le stock de fourrage a été augmenté. Ces ajustements ont permis de parvenir à une couverture des besoins en UF et en MAD.

\section{- Ajustements des PEF bovins à l'engraissement}

Dans les PEF bovins à engraisser réalisés, le recours aux aliments que l'on avait essayé de limiter lors de l'étape précédente a été finalement plus important que ce qui avait été prévu initialement. Les surfaces de cultures fourragères prévues ont été à peu près mises en place mais le recours aux fourrages a été moins important que prévu (parce que la production a été plus faible que prévue, la production de fourrage ayant été affectée sur des parcelles secondaires et le suivi de la culture ayant été négligé). Les producteurs ont préféré revenir aux pratiques d'alimentation habituelles à base de tourteau dans ce type de projet. Ainsi, les marges ont été moyennes, autour de 50000 FCFA par tête, mais elles auraient pu être améliorées si le recours aux aliments concentrés achetés avait été réduit.

\section{- Ajustements des PEF vaches laitières}

Dans les trois PEF vaches laitières réalisés, tous ont respecté les surfaces fourragères prévues, mais pour les aliments et le fourrage chaque producteur a procédé à des ajustements particuliers. $\mathrm{VL}_{1}$ s'est caractérisé par une augmentation forte du recours au fourrage et aux aliments, ce qui a abouti à une couverture des besoins alimentaires avec une marge de sécurité suffisante et à une marge par tête moyenne. $\mathrm{VL}_{2}$ s'est caractérisé, d'une part, par une réduction du recours au fourrage, une augmentation du recours aux aliments concentrés, ce qui a abouti à une meilleure couverture des besoins alimentaires par rapport au stade prévisionnel (avec une marge suffisante pour les UF), et, d'autre part, par une valorisation du lait brut en lait pasteurisé et en yaourts à la minilaiterie et sur le plan économique à une marge par vache élevée. $\mathrm{VL}_{3}$ ne s'est pas réalisé comme prévu (c'est-à-dire cinq vaches en lactation durant six mois). A la dernière minute, l'éleveur a décidé d'intégrer 12 vaches venant de mettre bas et, en conséquence, au bout de trois mois, après avoir réduit l'effectif à sept, les stocks d'aliment et de fourrage ont été épuisés, entraînant l'arrêt du projet. De plus l'éleveur a eu des problèmes de détournement de lait avec le berger, ce qui a expliqué les faibles quantités de lait vendues et le bilan économique négatif du projet.

\section{Evaluation des apprentissages}

La figure 3 montre que les apprentissages ont été plus importants chez les producteurs qui ont mené leur projet au bout $\left(\mathrm{PEF}_{\text {réalisés }}\right)$, notamment dans les domaines « connaissance des besoins des animaux » et « pratiques de cultures fourragères ». On a pu remarquer que dans les domaines de « l'amélioration des infrastructures d'élevage » et de la « gestion commerciale » les apprentissages ont été faibles sur l'ensemble de la population, ce qui n'est pas surprenant dans la mesure où dans cette phase expérimentale de la Capef le focus avait été davantage axé sur la maîtrise de l'alimentation. 


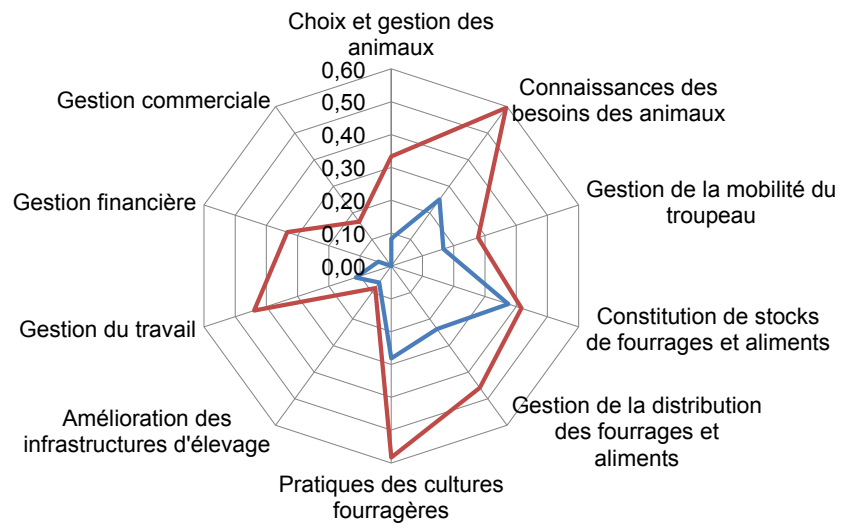

—PEF abandonnés au stade prévisionnel _ PEF réalisés

Figure 3 : radar des apprentissages selon le stade de réalisation du projet d'élevage familial dans les trois systèmes d'élevage étudiés dans l'ouest du Burkina Faso (PEF).

\section{DISCUSSION}

\section{Capef : démarche d'accompagnent ou de conception?}

Comment se positionne la Capef par rapport aux démarches d'accompagnement et de conception de systèmes d'élevage innovants ? La conception innovante nous a semblé être adaptée à notre problématique parce qu'elle vise à satisfaire des attentes nouvelles, pas nécessairement spécifiées au départ, et dont le chemin pour atteindre les objectifs visés n'est pas connu a priori et se précise à mesure que l'objet conçu prend forme (Le Masson et al., 2006 ; Meynard et Dourmad, 2014). En se référant à cette définition, la Capef n'est pas une démarche de conception de systèmes agricoles innovants proprement dite, car les PEF ne sont pas radicalement nouveaux et reposent sur des technologies connues par la plupart des producteurs de la zone d'étude. Néanmoins la Capef intègre bien une dimension de conception, pour le calibrage et l'amélioration du PEF au fur et à mesure des échanges avec le producteur. Elle s'inscrit donc dans l'esprit de la conception « pas à pas » de systèmes agricoles dans laquelle on cherche surtout à organiser le changement en s'appuyant sur des boucles d'apprentissages, où le producteur met au point son nouveau système progressivement, en même temps qu'il apprend à le piloter et réorganise son travail et ses moyens de production (Dedieu et al., 2011 ; Le Gal et al., 2011 ; Meynard et al., 2012 ; Meynard et Dourmad, 2014).

Aujourd'hui, dans le monde du développement rural, le terme « accompagnement » englobe une nébuleuse de pratiques visant le renforcement des capacités des individus et des organisations par l'apprentissage et l'acquisition de connaissances actionnables. La posture d'accompagnement correspond donc à un élargissement des fonctions du système de conseil aux producteurs (Faure et al., 2014). La Capef correspond aussi à une démarche d'accompagnement du producteur, dans la mesure où elle est basée sur un dialogue et un échange permanent entre le producteur et le conseiller tout au long du processus de préparation, de mise en œuvre et d'évaluation du PEF.

La Capef a été testée dans une optique d'accompagnement et de conseil individuel et n'a pas eu recours à des clubs de producteurs expérimentateurs comme dans le cas de la recherche action en partenariat (Vall et Chia, 2015). Il pourrait être intéressant d'introduire de tels clubs dans la Capef, la réflexion entre pairs étant une voie efficace pour apprendre et résoudre les problèmes auxquels on se confronte, d'autant plus que la Capef et la recherche action en partenariat sont proches dans leurs principes et dans leur appréhension de la construction du changement.

\section{Points forts de la Capef}

La Capef étant basée sur un dialogue permanent entre le producteur, porteur du PEF, et un conseiller en élevage, et sur des cycles de gestion " planification-action-bilan », permet à tout moment de prendre du recul sur le PEF, de déceler ses faiblesses, de réfléchir aux solutions possibles pour l'améliorer et de l'ajuster au fur et à mesure, et ainsi de parvenir à un projet plus réaliste sur le plan technique et économique qui est un objectif généralement recherché dans les démarches de conseil et d'accompagnement de l'élevage (Awono Bessa et al., 2003 ; Vall et al., 2007 ; Faure et al., 2014).

La Capef dépasse la simple aide à l'adoption de nouvelles techniques de production ou la simple vulgarisation, puisqu'elle développe les capacités des producteurs à construire des réponses aux problèmes, à travers la production de connaissances actionnables (Avenier et Schmitt, 2007) et de savoir-faire issus de boucles d'apprentissages (Kolb, 1984 ; Argyris et Schön, 2001). Les résultats montrent que la prise de distance sur les PEF permise par la Capef a eu des effets sur les pratiques des producteurs (choix des animaux, cultures fourragères, conduite de l'alimentation, comptabilisation des recettes et des dépenses) et sur leur stratégie (prévision des quantités de fourrages et d'aliments, calibrage du projet en fonction des ressources disponibles et des recettes escomptées, établissement d'un plan de trésorerie prévisionnel), témoignant d'apprentissages de première boucle. Mais la période d'accompagnement a sans doute été trop courte (6-12 mois) pour développer des apprentissages plus profonds (de deuxième boucle) chez les producteurs. Des itérations supplémentaires des PEF auraient été nécessaires pour que les producteurs fassent évoluer en profondeur leur système de valeurs en matière de rationnement des troupeaux (intégration des systèmes d'alimentation proposés par la zootechnie). Enfin, on peut aussi s’interroger sur la réalité de certains apprentissages révélés par l'enquête finale, au vu des ajustements réellement effectués et des résultats économiques médiocres obtenus par certains producteurs, ce qui nous conduit à aborder les faiblesses et les limites de la Capef.

\section{Points faibles de la Capef}

D'abord les résultats montrent que les calibrages et les ajustements des PEF n'ont pas toujours abouti aux résultats escomptés en termes de couverture des besoins des animaux (couverture avec une légère marge de sécurité), malgré la tendance à la convergence vers l'objectif de « $100 \%$ des besoins couverts » dans les PEF réalisés.

En matière d'alimentation, les producteurs font des choix qui prennent en compte d'autres critères que l'ajustement « parfait» des rations sur le plan de la nutrition animale, comme par exemple la disponibilité de la ressource sur l'exploitation ou sur le marché, la facilité d'utilisation, la disponibilité en terre pour des cultures fourragères. Ces autres critères doivent donc être recherchés et explicités lors de l'étape de calibrage du PEF, et si possible avant (durant le diagnostic).

Dans Altrop, le recours aux systèmes des UF et MAD a été privilégié pour l'instant, parce que c'est le système le plus utilisé localement par les zootechniciens et les conseillers en élevage, et le plus référencé dans les manuels de zootechnie à leur disposition. Mais à l'avenir il pourrait bien être remplacé par le système des unités fourragères lait (UFL), des unités fourragères viande (UFV) et des protéines digestibles dans l'intestin grêle (PDI), qui tend à devenir le nouveau système de référence (Jarrige, 1989).

Les aspects environnementaux ne sont pour l'instant pas pris en compte par la Capef, qu'il s'agisse des contributions positives des PEF à l'entretien de la fertilité des terres à travers la production de fumure organique (Vall et al., 2006), ou bien négatives comme les émissions de méthane (Herrero et al., 2008). Vu la montée des questions environnementales, il serait important d'intégrer ce volet dans 
la Capef, surtout si les PEF évoluent vers des projets de plus grandes dimensions, ce qui sera certainement le cas.

La Capef telle qu'elle a été expérimentée nécessite des visites d'exploitations très régulières, surtout au moment de la mise en œuvre des projets où une visite toutes les deux semaines était effectuée. Une fréquence aussi élevée était justifiée tant qu'il s'agissait d'un travail de recherche, mais dans une optique d'appui-conseil elle serait trop coûteuse et irréalisable. Il faudrait donc revoir le planning d'intervention du conseiller, ce qui nous conduit à la dernière partie de la discussion.

\section{Vers une application de la Capef?}

Dans l'étude présentée, le chercheur s'est substitué au conseiller, ce qui pose plusieurs questions si l'on se place dans une perspective d'utilisation de la démarche en conseil à l'élevage : en quoi la Capef est-elle mobilisable par des conseillers ? Ces conseillers existent-ils ? De quelles compétences ont-ils besoin ? Rappelons au préalable que le bilan des actions de conseil à l'élevage en Afrique subsaharienne, qui à ce jour demeurent des opérations pilotes, a montré qu'un accompagnement des producteurs dans la phase de conception et d'exécution d'activités innovantes ou de projets serait une garantie de leur réussite (Awono Bessa et al., 2003 ; McCown, 2003 ; Vall et al., 2007 ; Faure et al., 2014).

Dans la zone de l'étude et d'une manière générale en Afrique de l'Ouest (Club du sahel, 2008), l'appui à l'élevage est réalisé par des techniciens du ministère de l'élevage, qui interviennent principalement sur les aspects sanitaires et zootechniques, de façon prescriptive. Ces agents ne disposent pas de méthodes et d'outils pour accompagner les producteurs dans la préparation, la mise en œuvre et l'évaluation des projets de production et d'investissement, ou encore dans la gestion courante du troupeau et des différents ateliers. Donc la démarche Capef et les outils qu'elle utilise (Altrop) pourraient être mobilisés par ces agents de terrain, moyennant les adaptations discutées plus haut. Ils ont les connaissances techniques de base nécessaires, et aussi pratiques, étant eux-mêmes souvent éleveurs. Les compétences à renforcer à leur niveau concerneraient principalement la posture à adapter (compréhensive et didactique plutôt que prescriptive) et l'utilisation de l'outil informatique.

\section{CONCLUSION}

La démarche Capef vise à aider le producteur à réussir un projet d'élevage familial en l'ajustant progressivement, en même temps qu'il apprend à le piloter, se convainc de son intérêt, et réorganise progressivement le système d'alimentation du troupeau, le travail et la mobilisation des moyens de production. L'interaction permanente entre le producteur et le conseiller stimule la réflexion critique sur le projet d'élevage familial durant les étapes de diagnostic et de recherche d'options d'amélioration, ce qui permet de mieux ajuster l'offre alimentaire aux besoins et d'améliorer au final le bilan économique du projet. Vu les perspectives de développement de l'élevage en Afrique de l'Ouest et les difficultés des producteurs à mettre en œuvre leur projet individuel d'élevage, il est important de poursuivre le développement de ce type de démarche d'accompagnement des producteurs.

\section{Remerciements}

Cette étude a reçu le soutien financier du projet Options d'intensification durable (AusAid, Coraf) et une subvention de doctorat Aird/ Cirad. Les auteurs remercient les producteurs de Founzan, Gombéledougou et Koumbia qui leur ont permis de travailler avec eux tout au long de l'étude.

\section{REFERENCES}

Argyris C., Schön D., 2001. Apprentissage organisationnel. Théorie, méthode, pratique. De Boeck Supérieur "Management », Bruxelles, Belgique, $380 \mathrm{p}$.

Avenier M.J., Schmitt C., 2007. La construction de savoirs pour l'action. L'Harmattan, Paris, France, 245 p. (Coll. Action et savoir, sér. Rencontres )

Awono Bessa C., Havard M., Assana M., 2003. Le conseil à l'élevage : une démarche pour accompagner l'intégration de l'élevage à I'agriculture dans les exploitations agricoles du Nord-Cameroun ? In : Savanes africaines : des espaces en mutation, des acteurs face à de nouveaux défis (éds Jamin J.Y., SeinyBoukar L., Floret C.), mai 2002, Garoua, Cameroun. Prasac, N’Djamena, Tchad / Cirad, Montpellier, France

Bebe B.O., Udo H.M.J., Thorpe W., 2008. Characteristics of feeding and breeding practices for intensification of smallholder dairy systems in the Kenya highlands. Livest. Res. Rural Dev., 20 (2)

Bélières J.-F., Bonnal P., Bosc P.-M., Losch B., Marzin J., Sourisseau J.-M., 2014. Les agricultures familiales du monde. Définitions, contributions et politiques publiques. Cirad, Montpellier, France, 306 p. (Coll. A savoir)

Club du Sahel, 2008. Elevage et marché régional au Sahel et en Afrique de l'Ouest. Potentialités et défis. CSAO-OCDE, CDAO, Paris, France, $182 \mathrm{p}$.

Coulon J.B., Lecomte P., Boval M., Perez J.M., 2011. Numéro spécial, Elevage en régions chaudes. Prod. Anim., 24 (1) : 1-160

Dedieu B., Aubin J., Duteurtre G., Alexandre G., Vayssieres J., Bommel P., Faye B., 2011. Conception et évaluation de systèmes d'élevage durables en régions chaudes. Prod. Anim., 24 (1) : 113-128

Delgado C.L., 2003. Rising consumption of meat and milk in developing countries has created a new food revolution. J. Nutr., 133: 3907S-3910S

Delma B.J., Bougouma-Yaméogo V., Nacro H.B., Vall E., 2016a. Fragilité des projets d'élevage familiaux dans les exploitations de polycultureélevage au Burkina Faso. Cah. Agric., 25 (3), 35005, doi : dx.doi. org/10.1051/cagri/2016019

Delma B.J., Bougouma-Yameogo V., Nacro H.B., Vall E., 2016b. Altrop, un calculateur des rations des bovins pour I'accompagnement des projets d'élevage familiaux. Int. J. Biol. Chem. Sci., 10 (3) : 966-982.

FAO, 2009. La situation mondiale de l'alimentation et de l'agriculture. Le point sur l'élevage. FAO, Rome, Italie, 202 p.

Faure G., Havard M., Toillier A., Djamen Nana P., Moumouni I., 2014. Innovation dans les services de conseil aux exploitations agricoles familiales. In : Agriculture familiales, et mondes à venir (éd. Sourisseau J.M.). Quae, Paris, France, 257-267

Faye B., 2001. Role of animal husbandry to alleviate poverty [in French]. Rev. Elev. Med. Vet. Pays trop., 54 (3-4): 231-238

Herrero M., Thornton P.K., Kruska R., Reidr S., 2008. Systems dynamics and the spatial distribution of methane emissions from African domestic ruminants to 2030. Agric. Ecosyst. Environ., 126: 122-137

Jarrige R., 1989. Ruminant nutrition. Recommended allowances and feed tables. John Libbey, Paris, France, 389 p.

Kolb D.A., 1984. Experiential learning: Experience as the source of learning and development. Prentice Hall, Englewood Cliffs, NJ, USA, $22 \mathrm{p}$.

Le Gal P.Y., Dugue P., Faure G., Novaks., 2011. How does research address the design of innovative agricultural production systems at the farm level? A review. Agric. Syst., 104 (9): 714-728, doi:10.1016/j. agsy.2011.07.007

Le Masson P., Weil B., Hatchuel A., 2006. Les processus d'innovation: conception innovante et croissance des entreprises. Hermès Lavoisier, Paris, France

Lhoste P., Havard M., Vall E., 2010. La traction animale. Quae, CTA, PAG, Paris, France, 224 p. 
McCown R.L., 2003. Changing systems for supporting farmers' decisions: problems, paradigms, and prospects. Agric. Syst., 74: 179-220

Meynard J.M., Dedieu B., Bos A.P., 2012. Re-design and co-design of farming systems. In: An overview of methods and practices - farming systems research into the 21 st century: The new dynamic (Eds. Darnhofer I., Gibon D., Dedieu B.). Springer, Dordrecht, Netherlands, 407-432

Meynard J.M., Dourmad J.Y., 2014. L'innovation en élevage : de nouvelles démarches pour de nouveaux enjeux. Prod. Anim., 27 : $77-88$

Rivière R., 1991. Manuel de l'alimentation des ruminants domestiques en milieu tropical. IEMVT, Paris, France, 529 p. (Coll. Manuels et précis d'élevage)

Robinson T.P., Thornton P.K., Franceschini G., Kruska R.L., Chiozza F., Notenbaert A., Cecchi G., Herrero M., Epprecht M., Fritz S., You L., Conchedda G., See L., 2011. Global livestock production systems. FAO, Rome, Italy, $152 \mathrm{p}$.
Sow F.D., Aminata B., Maloney M., 2004. L'embouche paysanne, un exemple d'adaptation de l'élevage traditionnel à la nouvelle situation agricole dans le bassin arachidier du Sénégal. Cah. Agric., 13 : 211 219

Vall E., Chia E., 2015. Co-constructing innovation: action research in partnership. In: Family farming and the Worlds to come (éd. Sourisseau J.M.). Springer, Dordrecht, Netherlands, p. 237-253

Vall E., Djamen P., Havard M., Roesch M., 2007. Investir dans la traction animale : le conseil à l'équipement. Cah. Agric., 16 : 93-100, doi : 10.1684/agr.2007.0080

Vall E., Dugué P., Blanchard M., 2006. Le tissage des relations agriculture-élevage au fil du coton. Cah. Agric., 15 : 72-79

Zoundi J.S., Sawadogo L., Nianogo A.J., 2003. Pratiques et stratégies paysannes en matière de complémentation des ruminants au sein des systèmes d'exploitation mixte agriculture-élevage du plateau central et du Nord du Burkina Faso. Tropicultura., 21 : 122-128

\section{Summary}

Delma B.J., Nacro H.B., Bougouma-Yameogo V., Vall E. Participatory innovation design, and support of family livestock projects in Burkina Faso: explore, experiment, evaluate

In mixed farming systems in Western Burkina Faso, a large majority of farmers have a family livestock project (FLP) in mind. However, more than half of these projects do not even get started. Among those implemented, major flaws have been observed in livestock feed management, jeopardizing their profitability. This article presents an approach to design and support family livestock projects (DSFLP) conducted in partnership between the farmers and a consultant. This approach helps to assist farmers in preparing, implementing and assessing their projects. It was developed with 10 farmers whose projects concerned draft, fattening, or dairy cattle production. DSFLP involves four steps: i) diagnosis, ii) measurement and research on improvement options, iii) implementation and adjustment, and iv) assessment. In this approach, the farmers had gradually developed FLP, while learning to manage it, by reorganizing their work and means of production. DSFLP has enhanced their learning on i) the control of feeding practices and ii) developing livestock project strategies, to promote success. For the researcher, it helped to understand the rationality of farmers by participating in the construction and implementation of their projects.

Keywords: cattle, project design, family farm, animal feeding, participatory approach, Burkina Faso

\section{Resumen}

Delma B.J., Nacro H.B., Bougouma-Yameogo V., Vall E. Concepción participativa de innovaciones y acompañamiento de proyectos de cría familiar en Burkina Faso: explorar, experimentar y evaluar

Una gran mayoría de los policultores criadores del oeste de Burkina Faso tienen un proyecto de cría familiar (PCF) en mente. Sin embargo, más de la mitad de los proyectos no ven nunca la luz del día. Entre aquellos que se realizan, son observadas importantes debilidades a nivel de la gestión de la alimentación del ganado, lo que compromete su rentabilidad económica. El presente artículo presenta una guía de concepción y de acompañamiento del proyecto de cría familiar (CAPCF), llevado conjuntamente entre el productor y un consejero. Esta permite acompañar al productor durante la preparación, la ejecución y la evaluación de su proyecto. La guía fue elaborada con 10 productores que poseían PCF bovinos de tiro, bovinos de engorde o vacas lecheras en preparación. La CAPCF se desarrolla en cuatro etapas: a) diagnóstico, b) calibración e investigación de las opciones de mejoramiento, c) implementación y ajustamiento, y d) evaluación. Bajo este enfoque de concepción de acompañamiento, el productor estableció el PCF progresivamente, al mismo tiempo que aprendió a pilotearlo, reorganizando su trabajo y la asignación de sus medios de producción. La CAPCF desarrolló en los productores aprendizajes concernientes con el dominio de las practicas de alimentación y de las estrategias de elaboración de un proyecto de cría para mejorar el éxito. Para el investigador, esta permitió comprender el raciocinio de los criadores participando con ellos en la construcción y el desarrollo de la obra de sus PCF.

Palabras clave: ganado bovino, diseño de proyectos, explotación agrícola familiar, alimentación de los animales, enfoque participativo, Burkina Faso 
